\title{
Synthesis and Properties of a Novel Coating Material with High Thermal Conductivity and Insulation for Busbar
}

\author{
Yueju Zhao ${ }^{1, \text { a }}$, Runtao Dou ${ }^{2, b}$, Guogang Wang ${ }^{1, c}$, Dengxu Wang ${ }^{2, d^{*}}$ and \\ Shengyu Feng ${ }^{2, e}$ \\ ${ }^{1}$ Beijing Guodian Futong Science and Technology Development CO., LTD, Beijing 100070, China \\ ${ }^{2}$ Shandong University, Jinan 250100, China \\ azhaoyj@iccas.ac.cn, ${ }^{b} 506904194 @ q q . c o m,{ }^{c}$ ssnabi@126.com, ddxwang@sdu.edu.cn, \\ fsy@sdu.edu.cn
}

Keywords: Thermal Conductive Silicone Rubber; Aluminum Nitride; Thermal Conductive Materials Abstract. A novel coating material using polymethylvinyl silicone rubber as the base gum and aluminum nitride (AIN) as the thermal conductive filler was presented, and its mechanical property and thermal conductivity were measured. The results indicated that with increasing the content of AlN filled into the silicone rubber, the thermal conductivity, mechanical property and dielectric loss of the resulting heat conductive material increased while the dielectric strength decreased. And the effect of the particle size of AIN on thermal conductivity of filled silicone rubber showed that the thermal conductivity of silicone rubber filled with large size particle was better than that of silicone rubber filled with small size particle.

\section{Introduction}

Electric busbar has been widely used in the power supply system, including voltage switchgear, transformer and power panel since it plays an important role in the connection of lumped circuit switch and shunt circuitswitch ${ }^{[1-3]}$. To ensure the safe and stable operation of power system, electric bus is commonly covered by the insulation materials because it is easily affected by falling objects and natural weather, which could cause the busbar fault and even catch fire. Moreover, its temperature will rise due to electromagnetic induction and dielectric loss, resulting in reducing its mechanical property and dielectric property ${ }^{[4,5]}$. Therefore, the insulation materials must possess high heat resistance and good thermal conductivity.

At present, the main methodology of insulation protection for electric bus is using heat shrinkable materials and the most widely used insulating materials on the market is derived from epoxy resin ${ }^{[6,7]}$. Although epoxy resin based materials have excellent mechanical, electrical, chemical and adhesive performances, they have poor heat conductivity, low temperature curing, high temperature no-resistance and poor heat dissipation ${ }^{[8]}$. With the rapid development of industry, the power consumption and equipment capacity have largely increased. The epoxy resin based materials can not satisfy the needs of the long-term safe operation of the equipments. Therefore, a novel coating material with high thermal conductivity and insulation for busbar needs to be developed to ensure long-term reliable operation of the power equipments ${ }^{[9]}$.

Herein we presented a novel coating material using polymethylvinyl silicone rubber as the base gum and aluminum nitride (AIN) as the thermal conductive filler ${ }^{[10,11]}$. The resulting material exhibited high thermal conductivity and insulation. In addition, its mechanical property, thermal conductivity and dielectric property were also measured.

\section{Experimental}

Materials. Polymethylvinylsiloxane rubber $\left(M_{n} 5.8 \times 10^{5}\right.$; mole content of vinyl group, $\left.0.15 \%\right)$ was provided by Hesheng Zhejiang. The fumed silica (TS-530) with the specific surface area of $220 \mathrm{~m}^{2} / \mathrm{g}$ was purchased from Cabort. Aluminum nitride (AIN) was supplied by Sanhe Beijing, China. 
2,5-Bis(tert-butylperoxy)-2,5-dimethylhexane (DBPMH) was obtained from Akzo Nobel Peroxide, Tianjin, China.

Characterization. Thermal conductivity of silicone rubber was measured with a thermally conductive probe instrument RTC-C (Ron-ghua Electronic Instrument Manufacture, Jiangsu, China) under the stable state method. The size of the specimen is $13.0 \mathrm{~cm}$ in diameter and $0.20 \mathrm{~cm}$ in thickness.

Mechanical properties of silicone rubber, including tensile strength, hardness, and elongation at break were measured according to ASTM D412 and D624 procedures at a crosshead speed of 500 $\mathrm{mm} / \mathrm{min}$ using dumbbell-shaped test pieces in an electronic rubber tension tester (XLD-A, Second Experimental Machine Factory,Changchun, China) at room temperature. Testing of hardness was carried out using a Shore type A Durometer inaccordance with ASTM D2240.

Preparation of the coating material based on silicone rubber. The polymethylvinyl siloxane rubber was mixed with the silica TS-530 and aluminum nitride using a two-roll mill (SK-160, Shanghai Technical Research Institute of Light IndustryMachinery, China) at room temperature, then the curing agent DBPMH was added. The gross silicone rubber mixture was compression-molded at $160^{\circ} \mathrm{C}$ at the pressure of $10 \mathrm{MPa}$ for $25 \mathrm{~min}$. Then, the silicone rubbers were hung in the oven at $180^{\circ} \mathrm{C}$ for $2 \mathrm{~h}$ to post-cure.

Synthesis. The novel coating material was prepared using silicone rubber as the base gum, silica TS-530 as the reinforcing filler and AIN as the conductivity filler.

\section{Result and Discussion}

Thermal conductivity. Fig. 1 represents the thermal conduction of sillicone rubber filled with different amount of AIN. With the volume content of AIN increasing, the thermal conductivity of sillicone rubber was largely improved. When the volume content was low, the conductive fillers, i.e., AIN, in the dispersion system were isolated and thus the conductive chain cannot be formed. With the volume content increasing, fillers in the dispersion system were gradually contacted and conductive chain was formed. Thus the thermal conductivity was obviously enhanced.

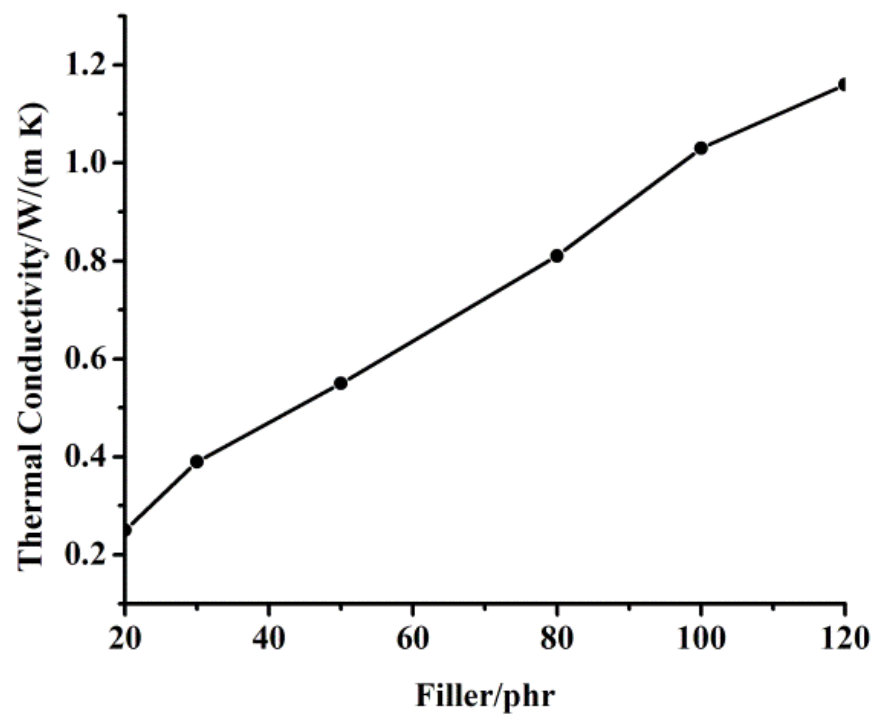

Fig. 1 Thermal conduction of silicone rubber filled with different amount of AlN

Mechanical properties. The mechanical properties of the rubber were shown in Fig. 2, Fig. 3 and Fig. 4. The hardness and tensile strength of the resulting silicone rubber were improved highly with the increase of the fillers, while the elongation at break of silicone rubber was decreased. It showed that the fillers had a reinforcing effect on the silicone rubber. Commonly, the fillers could be physically crosslinked with rubber molecules to make them closely integrated after the fillers evenly dispersing in the rubber matrix. It played the role of uniformly distributed load. Thus, the possibility of breaking the rubber was reduced and the mechanical properties of the rubber could be reinforced. 


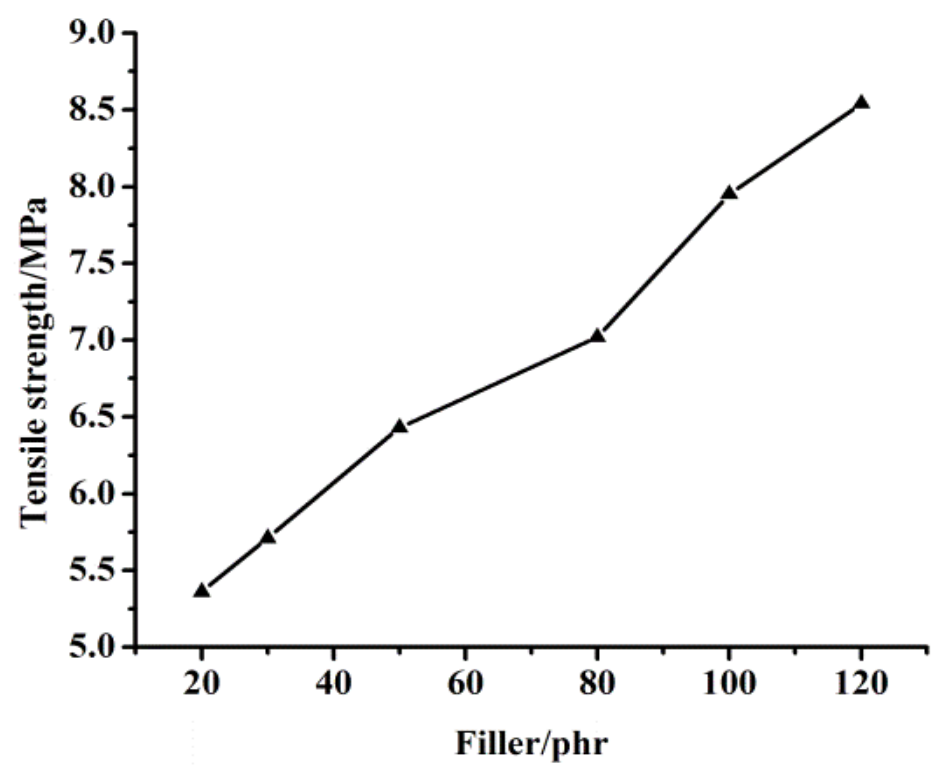

Fig. 2 Effect of AIN amount on tensile strength of filled silicone rubber

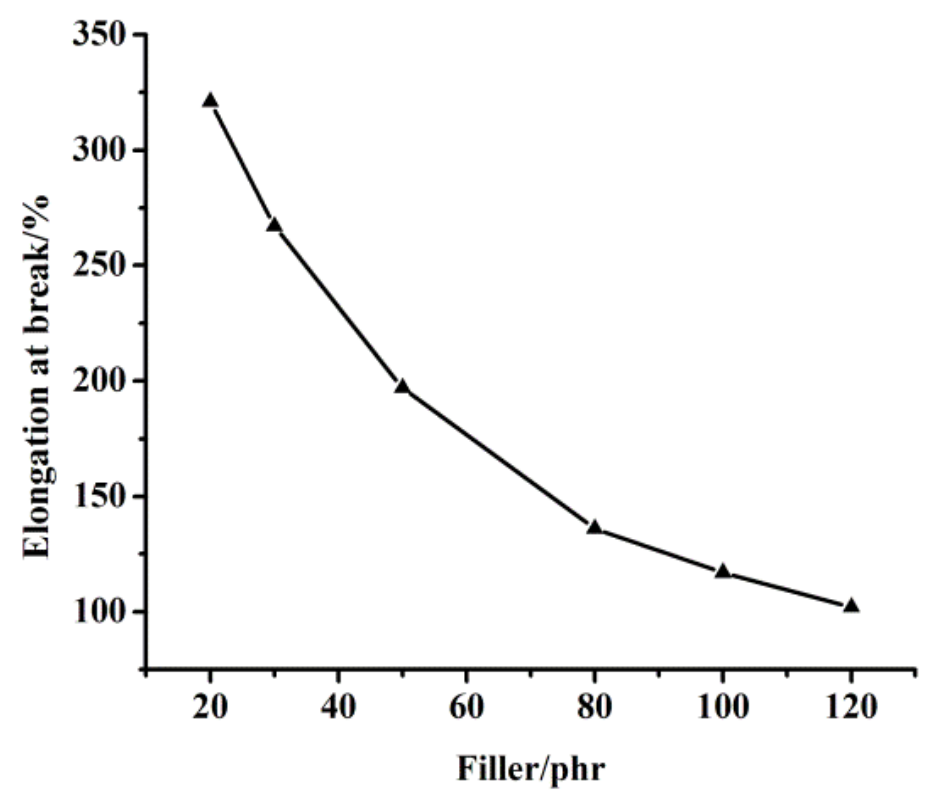

Fig. 3 Effect of AIN amount on the elongation at break of filled silicone rubber 


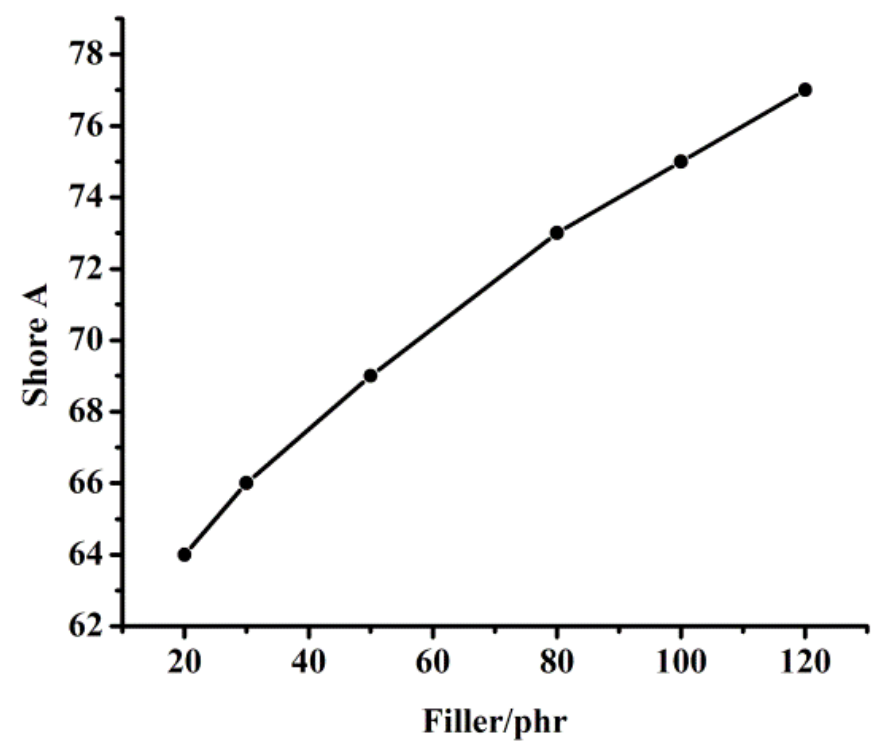

Fig. 4 Effect of AIN amount on hardness of filled silicone rubber

Dielectric properties. Fig. 5 showed the effect of AIN amount on dielectric loss of filled silicone rubber, the dielectric loss of filled silicone rubber increased with the increase of the AIN content. The effect of AIN amount on breakdown voltage of filled silicone rubber was indicated in Fig. 6. The breakdown voltage of filled silicone rubber reduced with the increase of fillers. The micropore in the rubber matrix increased with the increase of the fillers and the air in the micropore could decrease the density of the material. Thus, the dielectric properties of the silicone rubber would decrease.

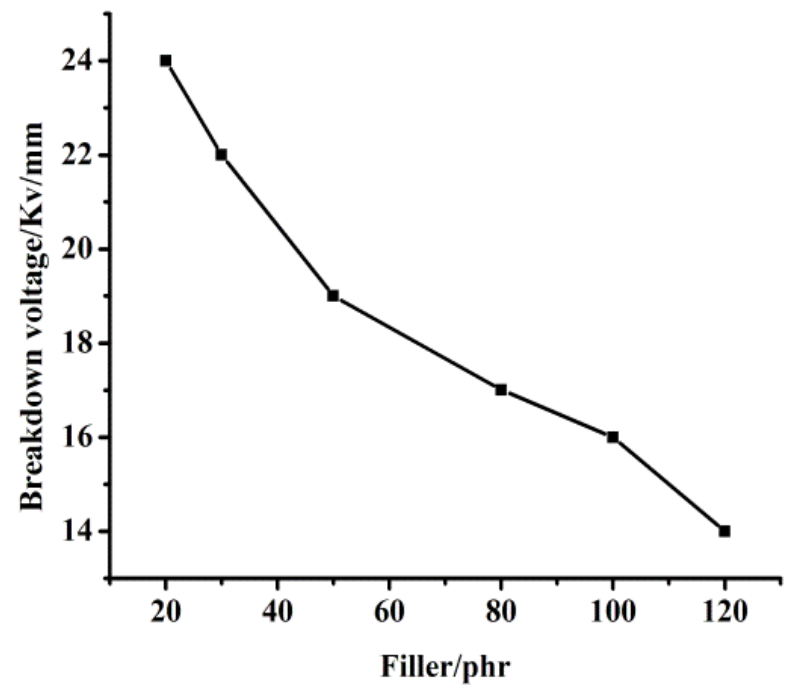

Fig. 5 Effect of AlN amount on breakdown voltage of filled silicone rubber 


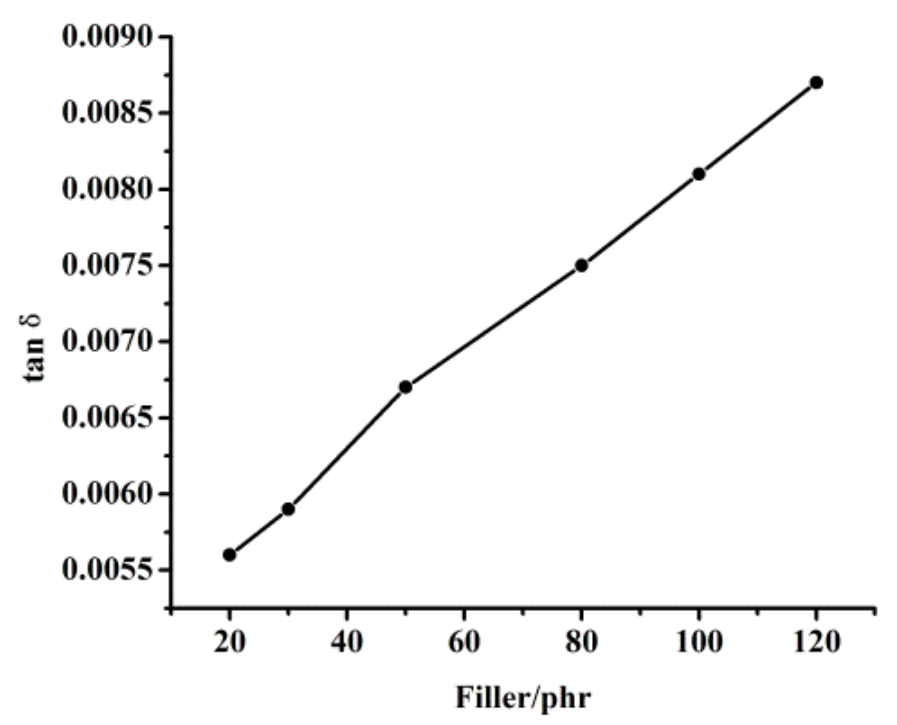

Fig. 6 Effect of AIN amount on dielectric loss of filled silicone rubber

The effect of thermal conductivity of silicone rubber filled with AIN with different particle size. Fig. 7 showed the effect of the particle size of AIN on thermal conductivity of filled silicone rubber. The thermal conductivity of silicone rubber filled with different size particle was highly improved with the increase of the fillers. When the amount of the fillers was less than $50 \mathrm{phr}$, the size of particle showed slight effect on the thermal conductivity because the fillers were surrounded by rubber matrix and equal thermal conductivity was achieved. On the contrast, when the amount was more than $50 \mathrm{phr}$, the thermal conductivity of silicone rubber filled with large size particle was better than that of silicone rubber filled with small size particle. As mentioned above, the conductive chain could be formed with the high content of fillers. Because the specific surface area of large size particle was smaller than small size particle, the phase interface area contacted with silicone rubber became smaller and enhanced the ability to form the thermal conductive path. Thus the thermal resistance became smaller, leading to higher thermal conductivity of silicone rubber filled with large size particle.

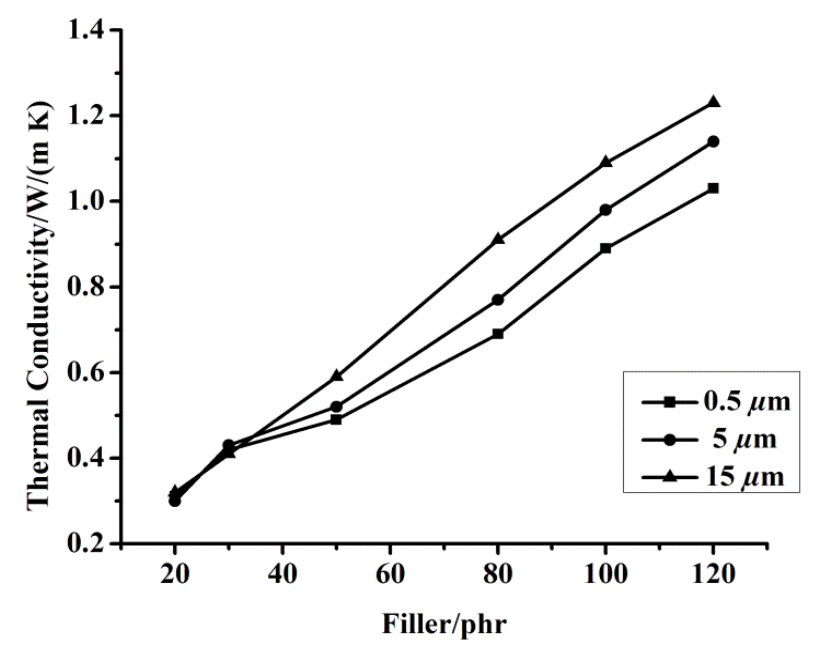

Fig. 7 Effect of particle size of AIN amount on thermal conductivity of filled silicone rubber

\section{Conclusions.}

A novel coating material with high thermal conductivity and insulation for busbar has been prepared using polymethylvinyl silicone rubber as the base gum and aluminum nitride (AIN) as the thermal conductive filler. The results showed that the mechanical property and thermal conductivity were increased with the increase of the fillers. The dielectric performance test showed that the dielectric loss of filled silicone rubber increased with the increase of the AIN content while the breakdown voltage of filled silicone rubber reduced. The coefficient of thermal conductivity test of silicone rubber with 
different size particles indicated that the thermal conductivity of silicone rubber filled with large size particle was better than the thermal conductivity of silicone rubber filled with small size particle.

\section{Acknowledgements.}

This work was supported by the Science and Technology project of the China State Grid Corp. (FTZX201404, Study on the development and the testing technology of the hybrid composite functional material for power transmission and transformation equipment).

\section{References}

[1] T. H. Hubing, J. L. Drewniak, T. P. VanDoren, and D. M. Hockanson, IEEE Trans. Electromagn. Compat., 37(1995) 155-166.

[2] G. N. Lester, E. Peters, IEEE Trans. Power Del., 11(1996) 1361-1366.

[3] W. Pfeiffer, IEEE. Electr. Insul. Mag., 17(2001) 34-47.

[4] Z. R. Li, S. H. Wang, Electr., 1(2013) 36-39.

[5] H. Ishida, S. Rimdusit, Thermochim. Acta, 320(1998) 177-186.

[6] Y. Ohki, IEEE. Electr. Insul. Mag., 26(2010) 48-49.

[7] D. A. Bolon, IEEE. Electr. Insul. Mag., 11(1995) 10-18.

[8] T. Takahashi, T. Okamoto, Y. Ohki, K. Shibata, IEEE Trans. Dielectr. Electr. Insul., 12(2005) 719-724.

[9] Y. P. Mamunya, V. V. Davydenko, P. Pissis, E. V. Lebedev, Eur. Polym. J., 38(2002) 1887-1897.

[10] Q. H. Mu, S. Y. Feng, Thermochim. Acta, 462(2007) 70-75.

[11] Y. W. Han, S. M. Lv, C. X. Hao, F. Ding, Y. Zhang, Thermochim. Acta, 529(2012) 68-73. 